\title{
ZINBIEL ALGEBRAS AND COMMUTATIVE ALGEBRAS WITH DIVIDED POWERS
}

\author{
IOANNIS DOKAS \\ Department of Mathematics and Statistics, University of Cyprus, P.O. Box 20537, CY-1678 Nicosia, Cyprus \\ e-mail:dokas@ucy.ac.cy
}

(Received 7 January 2009; accepted 10 June 2009; first published online 25 November 2009)

\begin{abstract}
In this paper, we prove that any Zinbiel algebra can be endowed with the structure of commutative algebra with divided powers. We introduce the notion of universal enveloping Zinbiel algebra of a commutative algebra with divided powers algebras. We prove that the free divided powers algebra on a free module $M$, is the divided powers sub-algebra generated by $M$, of the divided powers algebra induced by the free Zinbiel algebra on $M$. Finally, we construct a basis for the enveloping Zinbiel algebra.
\end{abstract}

2000 Mathematics Subject Classification. 17A32, 17A01, 13A99.

1. Introduction. The category dCom of commutative algebras with divided powers has been defined by H. Cartan in [2]. In particular it is proved in [2] that the homotopy of a commutative simplicial algebra is endowed with divided powers operations. Besides, we have to notice the contribution of N. Roby to the theory of commutative algebras with divided powers in [9-11]. In the theory of operads the category of commutative algebras with divided powers is the category of $\Gamma(\mathrm{Com})$ algebras, where Com denotes the commutative operad (for details see [3]). Moreover, the notion of commutative algebras with divided powers plays an essential role in the theory of crystalline cohomology. In particular, commutative divided powers algebras are used in order to overcome technical difficulties which arise in positive characteristic. In this way it is proved in [1], a crystalline Poincaré lemma analogue to Poincaré lemma in complex analytic geometry. Also, the structure of commutative algebras with divided powers appears in the calculation of Mac Lane homology of certain rings [5]. Moreover, we note that the notion of $p$-envelope is an important tool in the theory of $\mathrm{PD}^{1}$ differential operators developed by P. Berthelot and A. Ogus.

The category Zinb of Zinbiel algebras has been defined by J.-L. Loday in [4]. In connection with the notion of Koszul duality, Zinbiel algebras are Koszul dual to Leibniz algebras. A Zinbiel algebra is a commutative Dendiform algebra (for details, see [6]).

In this paper we prove that the category of Zinbiel algebras plays for the category of commutative algebras with divided powers a role analogue to this one of associative algebras for $p$-restricted Lie algebras. In particular, in Section 3 we prove that any Zinbiel algebra can be equipped with the structure of commutative algebra with divided powers. Thus, we construct the functor $\mathrm{dCom}_{\mathrm{C}}$ Zinb $\rightarrow \mathrm{dCom}$. As a consequence we obtain that the shuffle algebra has the structure of commutative algebra with divided powers. In Section 4 we introduce and we study the notion of Zinbiel enveloping

${ }^{1}$ The abbreviation PD it seems that comes from the french terminology puissances divisées. 
algebra $U_{z}(C)$ of commutative algebra with divided powers $C$. Moreover, we prove that the functor $U_{z}: \mathrm{dCom} \rightarrow$ Zinb is left adjoint to the functor ${ }_{\mathrm{dCom}}: \mathrm{Zinb} \rightarrow \mathrm{dCom}$. In Section 5, we prove that the free commutative divided power algebra $\Gamma(M)$ on a free $k$-module $M$ is the smallest divided powers sub-algebra of $(\operatorname{Zinb}(\mathrm{M}))_{\mathrm{dCom}}$ which contains $M$. Finally, in the last Section 6 we construct a $k$-basis for the enveloping Zinbiel algebra of a finite dimensional commutative algebra with divided powers.

2. Commutative algebras with divided powers. Let $k$ be a commutative ring. We recall the notion of commutative algebra with divided powers, for further details the reader may consult $[\mathbf{2}, \mathbf{9 - 1 1}]$. Let $A$ be an augmented commutative algebra over $k$, we will denote by $\bar{A}$ the augmentation ideal of $A$.

Definition 2.1. The unital algebra $(A, *)$ (or the non-unital algebra $\bar{A}$ ) is called an algebra with divided powers if there are, for $i>0$, operations $\gamma_{i}: \bar{A} \rightarrow \bar{A}$ such that the following relations hold:

$$
\begin{aligned}
\gamma_{1}(x) & =x, \quad \gamma_{i}(\lambda x)=\lambda^{i} \gamma_{i}(x), \quad \lambda \in k, \\
\gamma_{i}(x+y) & =\sum_{j=0}^{i} \gamma_{j}(x) * \gamma_{i-j}(y), \\
\gamma_{i}(x * y) & =i ! \gamma_{i}(x) * \gamma_{i}(y), \\
\gamma_{i}(x) * \gamma_{j}(x) & =\frac{(i+j) !}{i ! j !} \gamma_{i+j}(x), \\
\gamma_{i}\left(\gamma_{j}(x)\right) & =\frac{(i j) !}{i !(j !)} \gamma_{i j}(x),
\end{aligned}
$$

where by convention $\gamma_{0}(x):=1$ and $x, y \in \bar{A}$.

Definition 2.2. Let $A, B$ be commutative algebras with divided powers. A commutative algebra homomorphism $f: A \rightarrow B$ is called a homomorphism of divided powers algebras if $f \gamma_{i}=\gamma_{i} f$. We denote by dCom the category of divided powers algebras over $k$.

Definition 2.3. Let $C \in \mathrm{d} C$ om be a divided powers algebra. We call an ideal $I$ of $C$ divided ideal if for all $k>0$ and for all $\iota \in I$ we have $\gamma_{k}(\iota) \in I$.

REMARK 2.4. If $C$ is a divided powers algebra and $I$ a divided ideal of $C$, then the quotient algebra $C / I$ is endowed canonically with the structure of divided powers algebra.

REMARK 2.5. If the ring $k$ is a field of characteristic 0 any commutative graded algebra $A$ can be equipped with a unique system of divided powers given by

$$
\gamma_{i}(x):=x^{i} / i \text { ! where } x \in \bar{A}, \text { and } i>0 .
$$

REMARK 2.6. If the ring $k$ is a field of positive characteristic $p$, then by a theorem of H. Cartan in [2] we have that the system of divided powers on $A$ is induced by the operation $\gamma_{p}$. Moreover, let $A$ be an augmented commutative algebra together with a 
map $\pi: \bar{A} \rightarrow \bar{A}$ which verifies the relations of the $p t h$ divided power. Then by Theorem 1 in [12], $A$ is equipped with a unique system of divided powers with $\gamma_{p}=\pi$.

3. Zinbiel algebras. We remind that Zinbiel algebras have been introduced by J.-L. Loday in [4]. In particular in [4] is shown that Zinbiel algebras are Koszul dual to Leibniz algebras.

Definition 3.1. A Zinbiel algebra $(R, \prec)$ is a $k$-module equipped with a binary operation $\prec$ such that the following relation holds:

$$
(x \prec y) \prec z=x \prec(y \prec z)+x \prec(z \prec y)
$$

for all $x, y, z \in R$. We denote by Zinb the category of Zinbiel algebras over $k$.

REMARK 3.2. Any Zinbiel algebra $(R, \prec)$ can be considered with unit 1 by defining $\left(R^{+}, \prec\right)$, where $R^{+}:=k 1+R$ and for all $x \in R$ we define $1 \prec x=0$ and $x \prec 1=x$. We note that $1 \prec 1$ is not defined (for details, see [7]).

Any Zinbiel algebra $R$ is endowed with the structure of commutative algebra by defining a new operation $*$ for all $x, y \in R$ as $x * y:=x \prec y+y \prec x$. Therefore we have a functor Zinb $\rightarrow$ Com where Com denotes the category of commutative algebras over $k$.

Free Zinbiel algebras. It is shown in [4] that the free Zinbiel algebra over a $k$-module $V$ is the $k$-module $\bar{T}(V):=\oplus_{n \geq 1} V^{\otimes n}$ equipped with the product

$$
\left(x_{0} \ldots x_{p}\right) \prec\left(x_{p+1} \ldots x_{p+q}\right):=x_{0} s h_{p, q}\left(x_{1} \ldots x_{p+q}\right),
$$

where $s h_{p, q}$ is the sum over all $(p, q)$-shuffles. We will denote the free Zinbiel algebra on $V$ by $\operatorname{Zinb}(\mathrm{V})$.

Proposition 3.3. Let $(R, \prec)$ be a Zinbiel algebra over $k$. For $n>0$, we define $\gamma_{n}(x)$ inductively by $\gamma_{1}(x)=x$ and $\gamma_{n}(x)=x \prec \gamma_{n-1}(x)$, hence

$$
\gamma_{n}(x):=(\ldots(\underbrace{x \prec(x \prec(x \prec \ldots(x \prec x)}_{n} \ldots)) .
$$

Then we have the following relations:

$$
\begin{aligned}
\gamma_{n}(x) \prec x & =n \gamma_{n+1}(x) \\
x^{* n} & =n ! \gamma_{n}(x)
\end{aligned}
$$

for all $x \in R$ and $n \geq 1$.

Proof. Obviously relation (6) is true for $n=1$. Besides,

$$
\begin{aligned}
\gamma_{n+1}(x) \prec x & =\left(x \prec \gamma_{n}(x)\right) \prec x \\
& =x \prec\left(\gamma_{n}(x) \prec x+x \prec \gamma_{n}(x)\right) .
\end{aligned}
$$

Thus, by induction hypothesis we have

$$
\begin{aligned}
x \prec\left(\gamma_{n}(x) \prec x+x \prec \gamma_{n}(x)\right) & =x \prec\left(n \gamma_{n+1}(x)+\gamma_{n+1}(x)\right) \\
& =(n+1) \gamma_{n+2}(x) .
\end{aligned}
$$


For, relation (7) we easily see that for $n=1$ it is true. We suppose that is true for $n$, then

$$
\begin{aligned}
x^{* n+1} & =x^{* n} * x \\
& =n ! \gamma_{n}(x) * x \\
& =n !\left(\gamma_{n}(x) \prec x+x \prec \gamma_{n}(x)\right) .
\end{aligned}
$$

Therefore by relation (6) above we obtain that

$$
\begin{aligned}
x^{* n+1} & =n !(n+1) \gamma_{n+1}(x) \\
& =(n+1) ! \gamma_{n+1}(x) .
\end{aligned}
$$

THEOREM 3.4. Let $(R, \prec)$ be a Zinbiel algebra over $k$. Then $(R, *)$ is a commutative algebra with divided powers.

Proof. In order to prove the relations of the Definition 2.1 we proceed by induction. Obviously the relation (2) is true for $n=1$. We suppose that the relation is true for $n$ and by induction hypothesis we have,

$$
\begin{aligned}
(x & +y) \prec \gamma_{n}(x+y) \\
& =(x+y) \prec\left(\sum_{k=0}^{n} \gamma_{k}(x) * \gamma_{n-k}(y)\right) \\
& =\gamma_{n+1}(x)+\gamma_{n+1}(y)+x \prec \gamma_{n}(y)+y \prec \gamma_{n}(x)+(x+y) \prec\left(\sum_{k=1}^{n-1} \gamma_{k}(x) * \gamma_{n-k}(y)\right) .
\end{aligned}
$$

Besides,

$$
\begin{aligned}
(x+y) \prec\left(\sum_{k=1}^{n-1} \gamma_{k}(x) * \gamma_{n-k}(y)\right) & =\sum_{k=1}^{n-1}\left(\left(x \prec \gamma_{k}(x)\right) \prec \gamma_{n-k}(y)\right)+\sum_{k=1}^{n-1}\left(\left(y \prec \gamma_{n-k}(y)\right) \prec \gamma_{k}(x)\right) \\
& =\sum_{k=1}^{n-1}\left(\gamma_{k+1}(x) \prec \gamma_{n-k}(y)\right)+\sum_{k=1}^{n-1}\left(\gamma_{n-k+1}(y) \prec \gamma_{k}(x)\right) \\
& =\sum_{m=2}^{n}\left(\gamma_{m}(x) \prec \gamma_{n-m+1}(y)\right)+\sum_{k=1}^{n-1}\left(\gamma_{n-k+1}(y) \prec \gamma_{k}(x)\right) \\
& =\gamma_{n}(x) \prec \gamma_{1}(y)+\gamma_{n}(y) \prec \gamma_{1}(x)+\sum_{m=2}^{n-1}\left(\gamma_{m} * \gamma_{n-m-1}\right) .
\end{aligned}
$$

Now, the relation (3) is true for $n=1$. Suppose that the relation is true for $n$, we obtain

$$
(x * y) \prec \gamma_{n}(x * y)=n !(x \prec y+y \prec x) \prec\left(\gamma_{n}(x) * \gamma_{n}(y)\right) .
$$

Besides,

$$
\begin{aligned}
(x \prec y) \prec\left(\gamma_{n}(x) * \gamma_{n}(y)\right) & =\left((x \prec y) \prec \gamma_{n}(y)\right) \prec \gamma_{n}(x) \\
& =\left(x \prec \gamma_{n+1}(y)+x \prec\left(\gamma_{n}(y) \prec y\right)\right) \prec \gamma_{n}(x) .
\end{aligned}
$$


Thus using the relation (6) we get,

$$
\begin{aligned}
(x \prec y) \prec\left(\gamma_{n}(x) * \gamma_{n}(y)\right) & \left.=(n+1)\left(x \prec \gamma_{n+1}(y)\right) \prec \gamma_{n}(x)\right) \\
& =(n+1)\left(x \prec \gamma_{n}(x)\right) \prec \gamma_{n+1}(y) \\
& =(n+1)\left(\gamma_{n+1}(x) \prec \gamma_{n+1}(y)\right) .
\end{aligned}
$$

Therefore we have

$$
\begin{aligned}
(x * y) \prec \gamma_{n}(x * y) & =n !(n+1)\left(\gamma_{n+1}(x) \prec \gamma_{n+1}(y)+\gamma_{n+1}(y) \prec \gamma_{n+1}(x)\right) \\
& =(n+1) ! \gamma_{n+1}(x) * \gamma_{n+1}(y) .
\end{aligned}
$$

For the relation (4) we see that for $i=1$ and for all $j \in \mathbb{N}$, we have that

$$
\begin{aligned}
\gamma_{1}(x) * \gamma_{j}(x) & =x * \gamma_{j}(x) \\
& =x \prec \gamma_{j}(x)+\gamma_{j}(x) \prec x
\end{aligned}
$$

therefore by relation the (6) we obtain $\gamma_{1}(x) * \gamma_{j}(x)=(j+1) \gamma_{j+1}(x)$. We suppose that the relation is true for $i$ and for all $j \in \mathbb{N}$. We have

$$
\begin{aligned}
\gamma_{i+1} & (x) * \gamma_{j}(x) \\
& =\gamma_{i+1}(x) \prec \gamma_{j}(x)+\gamma_{j}(x) \prec \gamma_{i+1}(x) \\
& =\left(x \prec \gamma_{i}(x)\right) \prec \gamma_{j}(x)+\gamma_{j}(x) \prec\left(x \prec \gamma_{i}(x)\right) \\
& =x \prec\left(\gamma_{i}(x) * \gamma_{j}(x)\right)+\left(x \prec \gamma_{j-1}(x)\right) \prec \gamma_{i+1}(x) \\
& =x \prec\left(\gamma_{i}(x) * \gamma_{j}(x)\right)+x \prec\left(\gamma_{j-1}(x) \prec \gamma_{i+1}(x)+\left(x \prec \gamma_{i}(x)\right) \prec \gamma_{j-1}(x)\right) \\
& =x \prec\left(\gamma_{i}(x) * \gamma_{j}(x)\right)+x \prec\left(\gamma_{j-1}(x) \prec \gamma_{i+1}(x)\right)+x \prec\left(x \prec\left(\gamma_{i}(x) * \gamma_{j-1}(x)\right)\right) .
\end{aligned}
$$

Moreover,

$$
\begin{aligned}
\gamma_{j-1}(x) \prec \gamma_{i+1}(x) & =\left(x \prec \gamma_{j-2}(x)\right) \prec\left(x \prec \gamma_{i}(x)\right) \\
& =x \prec\left(\gamma_{j-2}(x) \prec\left(x \prec \gamma_{i}(x)\right)+\left(x \prec \gamma_{i}(x)\right) \prec \gamma_{j-2}(x)\right) \\
& =x \prec\left(\gamma_{j-2}(x) \prec \gamma_{i+1}(x)+x \prec\left(\gamma_{i}(x) * \gamma_{j-2}(x)\right) .\right.
\end{aligned}
$$

Therefore using induction hypothesis we get

$$
\begin{aligned}
\gamma_{i+1}(x) * \gamma_{j}(x) & =\left(\frac{(i+j) !}{i ! j !}+\frac{(i+j-1) !}{i !(j-1) !}+\cdots+\frac{(i+1) !}{i ! 1 !}+\frac{i !}{i ! 0 !}\right) \gamma_{i+1+j}(x) \\
& =\frac{(i+1+j) !}{(i+1) ! j !} \gamma_{i+1+j}(x) .
\end{aligned}
$$

Finally, for the relation (5) we easily see that for $i=1$ we have $\gamma_{j}(x)=(j ! / j !) \gamma_{j}(x)$ for all $j \in \mathbb{N}$. Suppose that the relation is true for $i$ and for all $j \in \mathbb{N}$ then we have

$$
\left.\left.\gamma_{i+1}\left(\gamma_{j}(x)\right)=\gamma_{j}(x) \prec(\underbrace{\gamma_{j}(x) \prec\left(\gamma _ { j } ( x ) \prec \left(\ldots \left(\gamma_{j}(x) \prec \gamma_{j}(x)\right.\right.\right.}_{i}) \ldots\right)\right) .
$$

Therefore by induction hypothesis we have

$$
\gamma_{i+1}\left(\gamma_{j}(x)\right)=\gamma_{j}(x) \prec\left(\frac{(i j) !}{i !(j !)^{i}} \gamma_{i j}(x)\right) .
$$


Moreover,

$$
\begin{aligned}
\gamma_{j}(x) \prec \gamma_{i j}(x) & =\left(x \prec \gamma_{j-1}(x)\right) \prec \gamma_{i j}(x) \\
& =x \prec\left(\gamma_{j-1}(x) * \gamma_{i j}(x)\right)
\end{aligned}
$$

thus by the relation (4) we have

$$
\begin{aligned}
\gamma_{i+1}\left(\gamma_{j}(x)\right) & =\frac{(i j) !}{i !(j !)^{i}} \frac{(j-1+i j) !}{(j-1) !(i j) !} \gamma_{i j+(j-1)+1}(x) \\
& =\frac{(i j) !}{i !(j !)^{i}} \frac{(j(i+1)) !}{j(i+1)(j-1) !(i j) !} \gamma_{(i+1) j}(x) \\
& =\frac{((i+1) j) !}{(i+1) !(j !)^{i+1}} \gamma_{(i+1) j}(x) .
\end{aligned}
$$

Let $V$ be a $k$-module. We consider an ordered basis $\mathcal{B}$ of $V$. We denote by $\mathcal{B}^{*}$ the free monoid on $\mathcal{B}$ and we put the lexicographical order $>$ on $\mathcal{B}^{*}$. Any element of $B^{*}$ is called a word. We recall that a word $l \in \mathcal{B}^{*}$ is called Lyndon word if it is smaller than all its non-trivial proper right factors. The tensor module $T(V)$ equipped with the shuffle product becomes a commutative algebra called shuffle algebra which we denote by $\left(T^{s h}(V), \star\right)$. (For details see $[\mathbf{8}]$.)

COROLlary 3.5. Let $V$ be a $k$-module. Then the shuffle algebra $\left(T^{s h}(V), \star\right)$ is a commutative algebra with divided powers $\gamma_{i}$. Moreover, if $k$ is a field of characteristic 0 then a $k$-basis for $\left(T^{s h}(V), \star\right)$ is given by the elements of the form:

$$
\gamma_{i_{1}}\left(l_{1}\right) \star \gamma_{i_{2}}\left(l_{2}\right) \cdots \star \gamma_{i_{n}}\left(l_{n}\right)
$$

where $l_{j}, j=1,2 \ldots, n$ are Lyndon words such that $l_{1}>l_{2}>\cdots>l_{n}$ and $i_{1}, i_{2}, \ldots, i_{n} \geq$ 1.

Proof. Let $\left(\mathrm{Zinb}(\mathrm{V})^{+}, \prec\right)$ be the free augmented Zinbiel algebra on the $k$ module $V$. From Theorem 3.2 above we have that $\operatorname{Zinb}(\mathrm{V})^{+}$is equipped with the structure of commutative algebra with divided powers $\gamma_{i}$. Moreover we note that for $v, w \in \operatorname{Zinb}(\mathrm{V})^{+}$we have $v \star w=v \prec w+w \prec v$. Therefore the divided powers algebra $\operatorname{Zinb}(\mathrm{V})_{\mathrm{dCom}}^{+}$is just the shuffle algebra $\left(T^{s h}(V), \star, \gamma_{i}\right)$. The Corollary follows easily from the Proposition 3.3 and the Theorem 6.1 in [8].

4. Universal enveloping Zinbiel algebra. From the Theorem 3.4 above we obtain a functor $-{ }_{\mathrm{dCom}}: \mathrm{Zinb} \rightarrow \mathrm{dCom}$. In particular, to each Zinbiel algebra $R \in$ Zinb we associate a divided powers algebra $R_{\mathrm{dCom}}$. In this section we will construct a left adjoint functor to the functor $-\mathrm{dCom}$. We will define the notion of enveloping Zinbiel algebra of a divided powers algebra.

Let $\left(C, *, \gamma_{i}\right) \in \mathrm{dCom}$ be a divided powers algebra. We consider the free Zinbiel algebra $\operatorname{Zinb}(\mathrm{C})$ on the $k$-module $C$. We denote by $J$ the Zinbiel ideal of $\operatorname{Zinb}(\mathrm{C})$ which 
is generated by the elements:

$$
\begin{aligned}
(x \prec y+y \prec x)-x * y, & \text { for all } x, y \in C \\
(\ldots(\underbrace{x \prec(\ldots(x \prec(x \prec x}_{i})) \ldots)-\gamma_{i}(x), & \text { for all } x \in C .
\end{aligned}
$$

Definition 4.1. Let $\left(C, *, \gamma_{i}\right) \in \mathrm{dCom}$ be a divided powers algebra. The Zinbiel algebra $U_{z}(C):=\operatorname{Zinb}(\mathrm{C}) / \mathrm{J}$ is called enveloping Zinbiel algebra of $C$.

Proposition 4.2. Let $C \in \mathrm{d}$ Com be a divided powers algebra and let $\sigma_{C}: C \rightarrow$ $U_{z}(C)$ be the natural map. Let $R \in Z i n b$ be a Zinbiel algebra. If $f: C \rightarrow R_{\mathrm{dCom}}$ is a divided powers algebras homomorphism then there exists Zinbiel homomorphism $\alpha$ : $U_{z}(C) \rightarrow R$ such that $\alpha \sigma_{C}=f$.

Proof. Since $f: C \rightarrow R$ is a $k$-linear map it can be extended to a unique Zinbiel homomorphism $\alpha^{\prime}: \operatorname{Zinb}(\mathrm{C}) \rightarrow \mathrm{R}$. Moreover, for all $x, y \in C$ we obtain:

$$
\begin{aligned}
\alpha^{\prime}(x \prec y+y \prec x-x * y) & =\alpha^{\prime}(x) \prec \alpha^{\prime}(y)-\alpha^{\prime}(y) \prec \alpha^{\prime}(x)-\alpha^{\prime}(x * y), \\
& =f(x) \prec f(y)-f(y) \prec f(x)-f(x * y), \\
& =0 .
\end{aligned}
$$

Besides, for all $x \in C$ we have

$$
\begin{aligned}
\alpha^{\prime}((\ldots(\underbrace{x \prec(\ldots(x \prec(x \prec x)}_{i}) \ldots) & \left.\left.=(\ldots \underbrace{\left(\alpha ^ { \prime } ( x ) \prec \left(\ldots \left(\alpha ^ { \prime } ( x ) \prec \left(\alpha^{\prime}(x) \prec \alpha^{\prime}(x)\right.\right.\right.\right.}_{i})\right) \ldots\right), \\
& =\underbrace{f(x) \prec(\ldots(f(x) \prec(f(x) \prec f(x))}_{i}) \ldots), \\
& =f\left(\gamma_{i}(x)\right), \\
& =\alpha^{\prime}\left(\gamma_{i}(x)\right) .
\end{aligned}
$$

Therefore the Zinbiel homomorphism $\alpha^{\prime}: \operatorname{Zinb}(\mathrm{C}) \rightarrow \mathrm{R}$ induces a unique Zinbiel homomorphism $\alpha: U_{z}(C) \rightarrow R$ such that $\alpha \sigma_{C}=f$.

COROllary 4.3. The functor $U_{z}: \mathrm{dCom} \rightarrow$ Zinb is left adjoint to the functor ${ }_{-\mathrm{dCom}}: \mathrm{Zinb} \rightarrow \mathrm{dCom}$.

Proof. Let $f: C \rightarrow R_{\mathrm{dCom}}$ be a divided powers algebras homomorphism. From Proposition 4.2 above we associate to $f$ a Zinbiel homomorphism $\alpha_{f}: U_{z}(C) \rightarrow R$. Let us denote by $\phi$ the map $\phi: f \mapsto \alpha_{f}$. Moreover, let $\alpha: U_{z}(C) \rightarrow R$ be a Zinbiel homomorphism. We associate to $\alpha$ a divided powers homomorphism $f_{\alpha}$ given by $f_{\alpha}:=\alpha \sigma_{C}$ where $\sigma_{C}: C \rightarrow U_{z}(C)$ is the natural map. If we denote by $\psi$ the map $\psi: \alpha \mapsto f_{\alpha}$ then we easily see that $\psi \phi=\phi \psi$. Therefore we get:

$$
H_{\operatorname{dom}}^{\mathrm{dCom}}\left(C, R_{\mathrm{dCom}}\right) \simeq \operatorname{Hom}_{\mathrm{Zinb}}\left(U_{z}(C), R\right) .
$$

5. Free divided powers algebras. Let $M$ be a $k$-module and let $\Gamma(M)$ the free divided powers algebra on $M$. We denote by $-^{[n]}: x \mapsto x^{[n]}$ the divided powers operations on $\Gamma(M)$. In [11], the tensor module $T(V)$ is equipped with the structure of 
divided powers algebra. We denote this structure by $(T(V), \circledast)$. Moreover, by Theorem 3 in [11] is proved that the module $(T S(M), \circledast)$ of symmetric tensors is divided powers sub-algebra of $(T(V), \circledast)$.

THEOREM 5.1. Let $M$ be a free $k$-module. The free divided powers algebra $\Gamma(M)$ on $M$ is the smallest divided powers sub-algebra of $\mathrm{Zinb}(\mathrm{M})_{\mathrm{dCom}}^{+}$which contains $M$.

Proof. Let $M$ be a free $k$-module with basis $\mathcal{B}=\left(e_{i}\right)_{i \in I}$. Since $M$ is a free $k$-module by the Proposition IV.5 in [10] there is a divided powers isomorphism $\psi: \Gamma(M) \rightarrow$ $T S(M)$ such that $\psi\left(x^{[n]}\right)=e^{\otimes n}$. Moreover, we have by the Proposition IV.5 in [10] that the elements of the form $e_{i_{1}}^{\otimes k_{1}} \circledast \cdots \circledast e_{i_{h}}^{\otimes k_{h}}$ form a $k$-basis for $(T S(M), \circledast)$.

We denote by $\left(F(M), \star, \gamma_{i}\right)$ the smallest powers sub-algebra of $\left(\operatorname{Zinb}(\mathrm{M})_{\mathrm{dCom}}^{+}, \star, \gamma_{\mathrm{i}}\right)$ which contains $M$. Moreover, we have

$$
\begin{aligned}
e_{i_{j}}^{\otimes k_{j}} & \left.=(\ldots \underbrace{e_{i_{j}} \prec \ldots e_{i_{j}} \prec\left(e_{i_{j}} \prec e_{i_{j}}\right.}_{k_{j}}) \ldots\right) \\
& =\gamma_{k_{j}}\left(e_{i_{j}}\right)
\end{aligned}
$$

We consider the $k$-linear map $\phi: T S(M) \rightarrow F(M)$ defined on basis elements by

$$
\begin{aligned}
\phi\left(e_{i_{1}}^{\otimes k_{1}} \circledast \cdots \circledast e_{i_{h}}^{\otimes k_{h}}\right): & =\gamma_{k_{1}}\left(e_{i_{1}}\right) \star \cdots \star \gamma_{k_{h}}\left(e_{i_{h}}\right) \\
& =e_{i_{1}}^{\otimes k_{1}} \star \cdots \star e_{i_{h}}^{\otimes k_{h}}
\end{aligned}
$$

We easily see using the relations of the Definition 2.1 that the $k$-linear map $\phi$ is actually a divided powers isomorphism. Therefore we obtain that the divided powers algebras $(T S(M), \circledast)$ and $(F(M), \star)$ are isomorphic.

Proposition 5.2. If $M$ be a free $k$-module, then we have:

$$
U_{z}(\bar{\Gamma}(M))=\operatorname{Zinb}(\mathrm{M}) .
$$

Proof. Since $M$ is free as a $k$-module from Proposition IV.5 in [10] we have that $\Gamma(M) \simeq T S(M)$ as divided powers algebras. Let $R$ be a Zinbiel algebra and let $f: \bar{\Gamma}(M) \rightarrow R_{\mathrm{dCom}}$ be a divided powers algebra homomorphism. We consider the restriction $f_{M}: M \rightarrow R$ of $f$ on $M$. The restriction $f_{M}$ is a $k$-linear map. Thus, from the universal property of $\operatorname{Zinb}(\mathrm{M})$ there is a unique Zinbiel homomorphism $\phi$ : $\operatorname{Zinb}(\mathrm{M}) \rightarrow \mathrm{R}$ such that $\phi i=f_{M}$, where $i$ denotes the natural map $i: M \rightarrow \operatorname{Zinb}(\mathrm{M})$. Moreover, from the Theorem 5.1 above the divided powers algebra $\bar{\Gamma}(M)$ is the divided powers sub-algebra of $\operatorname{Zinb}(\mathrm{M})_{\mathrm{dCom}}$ generated by $M$. Thus, we have an injection $j: \bar{\Gamma}(M) \rightarrow(\operatorname{Zinb}(\mathrm{M}))_{\mathrm{dCom}}$. Finally, we obtain that $\phi j=f$ and the universal Zinbiel enveloping algebra $\bar{\Gamma}(M)$ is $\operatorname{Zinb}(M)$.

Let $\left(C, C^{\prime}\right) \in \mathrm{dCom}$ be divided powers algebras and let $\phi: C \rightarrow C^{\prime}$ be a divided powers algebras homomorphism. We denote by $\sigma_{C}: C \rightarrow U_{z}(C)$ and $\sigma_{C^{\prime}}: C^{\prime} \rightarrow$ $U_{z}\left(C^{\prime}\right)$ the natural maps. We easily see that $\sigma_{C^{\prime}} \phi: C \rightarrow U_{z}\left(C^{\prime}\right)_{\mathrm{dCom}}$ is a divided powers homomorphism. Therefore there is a Zinbiel homomorphism $\bar{\phi}: U_{z}(C) \rightarrow U_{z}\left(C^{\prime}\right)$ such 
that the following diagram is commutative:

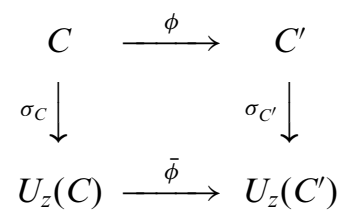

Let $C \in \mathrm{dCom}$ be a divided powers algebra and let $\sigma_{C}: C \rightarrow U_{z}(C)$ be the natural map. Moreover, let $I$ be a divided ideal of $C$. We denote by $\mathcal{R}$ the Zinbiel ideal of $U_{z}(C)$ generated by $\sigma_{C}(I)$.

Proposition 5.3. Let $C \in \mathrm{d} C o m$ be a divided powers algebra and let I be a divided ideal of $C$. Then we have a Zinbiel homomorphism $U_{z}(C / I) \simeq U_{z}(C) / \mathcal{R}$.

Proof. Following the previous notation we have the commutative diagram below:

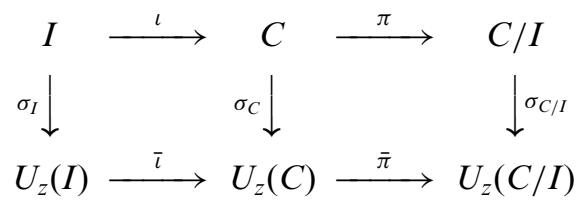

We can see from the commutative diagram that $\bar{\pi}\left(\sigma_{C}(I)\right)=0$. Therefore there is a Zinbiel homomorphism $\omega: U_{z}(C) / \mathcal{R} \rightarrow U_{z}(C / I)$ such that $\omega \psi=\bar{\pi}$ where $\psi$ : $U_{z}(C) \rightarrow U_{z}(C) / \mathcal{R}$ is the canonical homomorphism. Besides, we can easily see that the map $\psi \sigma_{C}: C \rightarrow U_{z}(C) / \mathcal{R}$ induce a divided powers homomorphism $\theta^{\prime}: C \rightarrow$ $\left(U_{z}(C) / \mathcal{R}\right)_{\mathrm{dCom}}$ such that $\theta^{\prime}(I)=0$. Therefore we get a divided powers homomorphism $\theta: C / I \rightarrow\left(U_{z}(C) / \mathcal{R}\right)_{\mathrm{dCom}}$ such that $\theta \pi=\psi \sigma_{C}$. From the universal property of the enveloping Zinbiel algebra, there is a Zinbiel homomorphism $\phi: U_{z}(C / I) \rightarrow U_{z}(C) / \mathcal{R}$ such that $\phi \sigma_{C / I}=\theta$.

Moreover, we have

$$
\begin{aligned}
\phi \omega \psi \sigma_{C} & =\phi \bar{\pi} \sigma_{C} \\
& =\phi \sigma_{C / I} \pi \\
& =\theta \pi \\
& =\psi \sigma_{C} .
\end{aligned}
$$

Thus we easily see that map $\phi \omega$ is the identity map on $U_{z}(C) / \mathcal{R}$. On the other hand, we have

$$
\begin{aligned}
\omega \phi \sigma_{C / I} \pi & =\omega \theta \pi \\
& =\omega \psi \sigma_{C} \\
& =\sigma_{C / I} \pi .
\end{aligned}
$$

Therefore, we obtain that the map $\omega \phi$ is the identity map on $U_{z}(C / I)$. In other words, we get an Zinbiel isomorphism $U_{z}(C) / \mathcal{R} \simeq U_{z}(C / I)$.

Corollary 5.4. Let $M$ be a free $k$-module and let $I$ be a divided ideal of $\bar{\Gamma}(M)$. Then we have a Zinbiel isomorphism $U_{z}(\bar{\Gamma}(M) / I) \simeq \operatorname{Zinb}(\mathrm{M}) / \mathcal{I}$ where $\mathcal{I}$ is the Zinbiel ideal of Zinb(M) generated by $I$. 
Proof. It follows easily from the Propositions 5.2 and 5.3 above.

6. Standard basis. Let $\left(C, *, \gamma_{i}\right)$ be a divided powers algebra with an ordered basis $B=\left\{e_{1}, e_{2}, \ldots, e_{n}\right\}$. We suppose that $e_{n}>e_{n-1}>\cdots>e_{2}>e_{1}$ and we endow $\operatorname{Zinb}(\mathrm{C})$ with the lexicographic order $>$. Moreover, we define a total order $\lessdot$ in $\operatorname{Zinb}(\mathrm{C})$ as follows:

$$
w \lessdot v=\left\{\begin{array}{l}
|w|<|v|, \\
|w|=|v|, \text { and } w>v .
\end{array}\right.
$$

where $w, v \in \operatorname{Zinb}(C)$ and $|w|$ denotes the length of the word $w \in \operatorname{Zinb}(C)$.

REMARK 6.1. We remark that the total order $\lessdot$ has the property that for a given word $w \in \operatorname{Zinb}(\mathrm{C})$ we have a finite number of words $v \in \operatorname{Zinb}(\mathrm{C})$ such that $v \lessdot w$.

Definition 6.2. We call a word $w \in \operatorname{Zinb}(\mathrm{C})$ standard if $w$ cannot be written as $k$-linear combination of smaller words modulo the ideal $J$. We denote by $S$ the set of the cosets in $U_{z}(C)$ of the standard words.

Proposition 6.3. Let $C \in \mathrm{d} C o m$ be finite dimensional divided powers algebra. The set $S$ is a basis for the enveloping Zinbiel algebra $U_{z}(C)$.

Proof. Let $w \in \operatorname{Zinb}(\mathrm{C})$ be a word. If $w$ is not standard then we can write $w$ as a linear combination of smaller words modulo the ideal $J$. If one of these words is not standard the we can write it as a linear combination of smaller words modulo $J$. From the Remark 6.1 above this process is finite. Therefore the set $S$ generates $U_{z}(C)$.

Moreover, we prove by contradiction that the set $S$ is linear independent. Suppose that there is modulo $J$ a non-trivial linear relation among standard words. Then we could write modulo $J$ the maximal word as a linear combination of smaller words. Therefore, the maximal word would not be standard.

The next proposition shows that the property of a word being standard is not trivial.

Proposition 6.4. The words $w$ of the form $w=e_{i_{1}} \otimes e_{i_{2}} \otimes \cdots \otimes e_{i_{n}}$, where $i_{1}<i_{2}<$ $\cdots<i_{n}$ are not standard.

Proof. We recall that $w=\left(\ldots\left(e_{i_{1}} \prec\left(e_{i_{2}} \prec \ldots \prec\left(e_{i_{n-1}} \prec e_{i_{n}}\right) \ldots\right)\right.\right.$. Besides, we have

$$
\begin{aligned}
e_{i_{j}} \star e_{i_{k}} & =e_{i_{j}} \prec e_{i_{k}}+e_{i_{k}} \prec e_{i_{j}} \\
& =e_{i_{j}} * e_{i_{k}} \quad \bmod J .
\end{aligned}
$$

Moreover for $e_{i_{j}}, e_{i_{k}}, e_{i_{h}} \in B$ we have,

$$
\begin{aligned}
e_{i_{j}} \star e_{i_{k}} \star e_{i_{h}} & =\left(e_{i_{j}} * e_{i_{k}}+j\right) \star e_{i_{h}} \bmod J \\
& =\left(e_{i_{j}} * e_{i_{k}}\right) \star e_{i_{h}} \bmod J \\
& =e_{i_{j}} * e_{i_{k}} * e_{i_{h}} \quad \bmod J
\end{aligned}
$$

where $j \in J$. Therefore we get

$$
e_{i_{1}} \star e_{i_{2}} \star \cdots \star e_{i_{n}}=e_{i_{1}} * e_{i_{2}} * \cdots * e_{i_{n}} \bmod J .
$$


We now remark that when we develop the quantity $e_{i_{1}} \star e_{i_{2}} \star \cdots \star e_{i_{n}}$ we get

$$
e_{i_{1}} \star e_{i_{2}} \star \cdots \star e_{i_{n}}=w+\sum_{v \in \operatorname{Zinb}(\mathrm{C})} v,
$$

where the words $v \in \operatorname{Zinb}(\mathrm{C})$ are such that $|v|=|w|$ and $v>w$. The left-hand side of the equation (8) belongs to $C$ modulo the ideal $J$. On the right-hand side of the equation (8) the words $v$ appearing in the sum are less than $w$ with respect to the total order $\lessdot$. Therefore, we see that the word $w$ can be written as a linear combination of smaller words modulo $J$.

\section{REFERENCES}

1. P. Berthelot and Arthur Ogus, Notes on Crystalline Cohomology, Annals of Mathematics Studies (Princeton University Press, 1978).

2. H. Cartan, Algèbres de Eilenberg-MacLane et homotopie, Seminaire Henri Cartan, 7ème année 1954-1955, 2ème éd. Ecole Normale Supérieure, Paris (1956).

3. B. Fresse, On the homotopy of simplicial algebras over an operad, Trans. Amer. Math. Soc. 352 (2000), 4113-4141.

4. J.-L. Loday, Cup-product for Leibniz cohomology and dual Leibniz algebras, Math. Scand. 77 (1995), 189-196.

5. J.-L. Loday, Cyclic Homology, Grundlehren der Mathematischen Wissenschaften, 301 (Springer-Verlag, Berlin, 1998).

6. J.-L. Loday, Dialgebras, in Dialgebras and related operads, Springer Lecture Notes in Math. 1763 (2001), 7-66.

7. J.-L. Loday, Scindement d' associativité et algèbres de Hopf. Proceedings of the colloquium dedicated to the memory of Jean Leray, Nantes, France, June 17-18, 2002. Paris: Societe Mathematique de France. Seminaires et Congres 9, 155-172 (2004).

8. C. Reutenauer, Free Lie algebras, London Math. Soc. Monos. (N.S), No. 7 (Oxford Univ. Press, 1993).

9. N. Roby, Les algèbres à puissances divisées, Bull. Sc.math. Série 2, t. 89 (1965), 75-91.

10. N. Roby, Lois polynomes et lois formelles en théorie des modules, Annales scientifiques de l' E.N.S. 3 serie, tome 80, num. 3 (1963), 213-348.

11. N. Roby, Construction de certain algèbres à puissances divisées, Bull. Soc. Math. France 96 (1968), 97-113. 523-529.

12. J.-P. Soublin, Puissances divisées caractéristique non nulle, Journal of Algebra 110 (1987), 